\title{
Effect of Bioaugmentation by Using Cow Manure Microbial Consortium on Microbial Communities for Treating Food Waste with Anaerobic Digestion
}

\author{
Nadya Y. Fahmi ${ }^{1}$, Cindy R. Priadi ${ }^{2 *}$, and Heri Hermansyah ${ }^{3}$ \\ ${ }^{1,2}$ Civil and Environmental Engineering Department, Faculty of Engineering, Universitas Indonesia, \\ Depok, 16424, Indonesia \\ ${ }^{3}$ Chemical Engineering Department, Faculty of Engineering, Universitas Indonesia, Depok, 16424, \\ Indonesia
}

\begin{abstract}
.
The number of microorganisms is the key factor affecting biodegradation capacity for anaerobic digestion (AD). Bioaugmentation strategy using an enriched culture has been known give supplementary microorganisms and consequently improve the AD performance, however bioaugmentation using a microbial consortium without enrichment for treating food waste (FW) is unknown. In the present study investigated whether bioaugmentation can improve the $\mathrm{AD}$ performance of $\mathrm{FW}$, as well as the effect of addition microbial consortium on the microbial community. This pilot scale study using stirrer-less reactors made of LLDPE, with the total and effective volume of $462 \mathrm{~L}$ and $369 \mathrm{~L}$, respectively. Bioaugmentation seed (BS) was a cow manure microbial consortium collected from slaughterhouse in Curug, Depok and the frequency of bioaugmentation was performed in every three days. The microbial community was examined using metagenomic next-generation sequencing (NGS). The effect of bioaugmentation using BS without enrichment was revealed as well. Findings demonstrated that effectively increase biogas production $(201 \mathrm{~L} /$ day $)$ and methane yield (546 L/kg VS $\mathrm{VSded}_{\text {add }}$ ), compared to non-bioaugmentation $(66 \mathrm{~L} /$ day) and (296 L/kg VS added . The increased biogas production and methane yield shows the feasibility of applying cow manure (CM) for bioaugmentation in $\mathrm{AD}$ of $\mathrm{FW}$. Furthermore, NGS analysis showed that BS enhanced the population of Methanosarcina and Methanosaeta. The abundance at $17.29 \%$ and $11.29 \%$, respectively. The higher Methanosarcina and Methanosaeta indicate the acetoclastic pathway was dominant on methanogenesis. The success of bioaugmentation using $\mathrm{CM}$ depends on environmental factors suitable for microbial life.
\end{abstract}

Keywords: Anaerobic Digestion, Bioaugmentation, Cow manure, Food Waste and Microbial community 


\section{Introduction}

Indonesia waste generation in 2020 is around 67.8 million tons. There are $60 \%$ of organic waste, $15 \%$ plastic waste, $10 \%$ paper waste, and $15 \%$ other waste. The organic waste dominated by FW (KLHK, 2020). Food waste is often mixed with other wastes and disposed such as landfilling and open burning. It would cause serious problems including health risk, environmental contamination such as greenhouse gas emissions, water and air pollution, etc (Schanes et al., 2018). Existing problems encourage the application of anaerobic digestion (AD). AD is known to be one of the best technologies for treating FW because it has suitable characteristics, i.e., macromolecular composition (e.g., sugar, proteins etc.), high level of biodegradability and a high volatile solid (VS) content (Fisgativa et al., 2016). AD can produce methane from the degradation of organic materials in FW as sustainable energy and environmental friendly thereby reducing greenhouse gas emissions, dependence on fossil fuels, the volume of solid waste and waste disposal costs (Vögeli et al., 2014).

The number of microorganisms is the key factor affecting biodegradation capacity. Thus a fine balance between the microbial communities which carry out hydrolysis, acidogenesis, acetogenesis and methanogenesis steps, as well as the nutrients provided for a successful digestion process to provide the highest conversion rate of organic material into methane (Negri et al., 2020). In maintaining a fine balance of the microbial communities, bioaugmentation has been suggested to recover systems efficiency or increase the performance of $\mathrm{AD}$ due to the higher microbial diversity and abundance (Romero-Güiza et al., 2016). Bioaugmentation aims to enhance AD performance by adding functional microorganisms. However, bioaugmentation relies on the abilities of the injected bacterial community to system (Sharma and Melkania, 2018). Mehariya et al (2018) also mentioned that bioaugmentation can be used to enhance the microbial community structure of the digester which increases biogas production.

Each microbe has their substrate specificity and capability to adapt environments as well as preferred pathways. Bioaugmentation culture could be individual strain or mixed culture which can be selected according to the goal and compatibility of the process (Mehariya et al., 2018). Tsapekos et al (2017) argued that pure microbe culture has a drawback that are too ideal, making them vulnerable in a bioreactor containing a complex microbial consortium. Therefore, choosing the mixed culture for bioaugmentation might be more advantageous as the introduced microbial consortium could provide a metabolic diversity and robustness to survive in a bioreactor containing an established indigenous community (Li et al., 2020c). As the previous studies reported, bioaugmentation has been successfully increase AD performance using enriched culture from full-scale anaerobic digesters ( $\mathrm{Li}$ et al., 2018a; Li et al., 2018b; Jiang et al., 2020). However, limited knowledge in previous research about bioaugmentation of pilot-scale for treating FW. In addition, the challenge that becomes the problem is Indonesia does not yet have many full-scale digester units to use sludge as a enriched culture so it must be use the other alternative for bioaugmentation culture.

The potential of CM for bioenergy production (especially biogas) have been reported in several studies to produce methane yield about 55\% (Oladejo et al., 2020), to 
produce the highest methane ( $\left.227 \mathrm{~mL} / \mathrm{g} \mathrm{VS}_{\text {added }}\right)$ as an inoculum in degrading $\mathrm{FW}$ (Dhamodharan et al., 2015). Moreover, used as a enriched bioaugmentation culture from CM digester to degrade complex organic matter ( $\mathrm{Li}$ et al., 2020c). However, how to evaluate the feasibility of bioaugmentation in pilot-scale AD using CM without enrichment is unknown. Hence, this study aims (1) to inoculate CM without enrichment for enhance $\mathrm{AD}$ performance of $\mathrm{FW}$; and (2) to evaluate the change microbial community before and after bioaugmentation.

\section{Materials and Methods}

\subsection{Substrate, inoculum and bioaugmentation culture}

The food waste used in present study was collected from the canteen in Al-Hikam Islamic Boarding School Depok, Indonesia. FW was sorted from bones, fruit skins, and seeds. FW was kept under iced conditions at $4{ }^{\circ} \mathrm{C}$ before use. The inoculum used in this study is mixture of $\mathrm{CM}$ and water. $\mathrm{CM}$ is diluted with water in a ratio of $1: 1$. The BS was also a cow manure microbial consortium diluted with water in ratio $1: 3$ and finally do the stirring to be homogeneous. The $\mathrm{CM}$ was taken from slaughterhouse in Curug, Depok, Indonesia. Basic information of substrate, inoculum and BS is listed in Tab 1. AD category in this study is dry system (> 15\% TS) (Gong et al., 2020).

Table 1. Characteristics of substrate, inoculum, and bioaugmentation seed

\begin{tabular}{|l|c|c|c|}
\hline \multicolumn{1}{|c|}{ Parameter } & Substrate & Inoculum & BS \\
\hline $\mathrm{pH}$ & $4.7 \pm 0.3$ & $7.3 \pm 0.1$ & $6.2 \pm 0.2$ \\
\hline TS (\% wt) & $22.8 \pm 0.3$ & $6.87 \pm 0.5$ & $2.74 \pm 0.34$ \\
\hline VS (\% TS) & $95.0 \pm 0.3$ & $88.37 \pm 4.7$ & $89.04 \pm 3.57$ \\
\hline
\end{tabular}

\subsection{Experimental procedures}

This pilot scale study using stirrer-less reactors made of LLDPE, with the total and effective volume of $462 \mathrm{~L}$ and $369 \mathrm{~L}$, respectively. The semi-continuous $\mathrm{AD}$ experiment for FW was operated at a mesophilic condition $\left(27.3 \pm 1{ }^{\circ} \mathrm{C}\right)$ with 5 feedings weekly (from Monday to Friday and no feeding over the weekends). The OLR was set at $30 \%$ of $1.5 \mathrm{~kg} \mathrm{VS} / \mathrm{m}^{3} /$ day. The effect of bioaugmentation was investigated in the same reactor in series. Operational without bioaugmentation has been operated for 32 days (day 1 to 32). From day 33 to 54, the reactor received BS with frequency and dosage were performed in every three days and $0.25 \mathrm{~g} \mathrm{VS} / \mathrm{L} /$ day, respectively.

\subsection{Analytical methods}

The $\mathrm{pH}$ value was recorded by probe (HI-98107). Total solids (TS) and volatile solids (VS) were drawn through standard method (APHA, 2017). Biogas volume was measured using vacuum pump and flowmeter, then $1 \mathrm{~L}$ of biogas was extracted to tedlar bag (Zefon International, Inc) every two days to analyze the methane content by a gas chromatography (GC-2014) with a thermal conductivity and helium as the carrier gas. The value of total alkalinity (TA) was determined by titration. Volatile fatty acid as acetic acid (VFA) was determined via distillation (APHA, 2017). 


\subsection{Microbial analysis}

Samples were taken on last day of without and with bioaugmentation stage for DNA extraction. Total genome DNA from samples was extracted using CTAB/SDS method. DNA concentration and purity was monitored on $1 \%$ agarose gels. According to the concentration, DNA was diluted to $1 \mathrm{ng} / \mu \mathrm{L}$ using sterile water. The microbes in the samples were characterized by employing $16 \mathrm{~S}$ rDNA gene sequencing. For identifying microbial community, 341F (50-CCTAYGGGRBGCASCAG-30) and 806R (50-GGACTACNNGGGTATCTAAT-30) were the bacteria primer pair for amplification with hypervariable area V3 - V4. PCR reactions were carried out with Phusion ${ }^{\circledR}$ High-Fidelity PCR Master Mix (New England Biolabs). The mixed PCR products were purified with Qiagen Gel Extraction Kit (Qiagen, Germany). The libraries generated with NEBNext® UltraTM DNA Library Prep Kit for Illumina and quantified via Qubit and Q-PCR, then analysed by Illumina platform. Paired-end reads were merged using FLASH (V1.2.7).

\subsection{Statistical Analysis}

A Wilcoxon test was performed using SPSS (Version 21.0), and Asymp.sig (2-tailed) $<0.05$ were considered significant.

\section{Results and Discussion}

\subsection{Biogas production and methane yield}

The daily biogas production and methane yield are shown in Fig. 1. Bioaugmentation was successfully increased biogas production 3-fold (average of 201 L/day), compared to non-bioaugmentation (average of $66 \mathrm{~L} / \mathrm{day}$ ). The first peak value of $94.15 \mathrm{~L} /$ day on day 12. During bioaugmentation, the volume of biogas tends to fluctuate. Gas peak is caused by adding of BS every three days. The first peak (212.05 L/day) on day 36 , followed by second and third peak value $302.35 \mathrm{~L} /$ day and 242.50 L/day on day 47 and 52, respectively. The peak observed with bioaugmentation could be attributed by a shorter lag phase, the availability of easily degraded organic matter in the substrate (Aragaw et al., 2013). Added microorganisms have increased the microbial structure to boost the methanogenesis ( $\mathrm{Li}$ et al., 2020c). Moreover, statistical analysis (Tab 2) was showed the sig value. $<0.05$ which indicated that the bioaugmentation significantly affects to higher biogas production. To summarize, we demonstrated that bioaugmentation with microbial consortium from $\mathrm{CM}$ without enrichment have positive influence on FW treatment to produce biogas.

Figure 1: Daily biogas production and methane yield during operational AD 


\section{$7^{\text {th }}$ International Conference On}

Modern Approaches in SCIENCE,

TECHNOLOGY \& ENGINEERING

\section{8-20 June, 2021}

Brussels, Belgium

By comparing the methane yield between process with and without bioaugmentation, we observed that the enhanced average of methane yield about 45,8\% (546 L/kg $\mathrm{VS}_{\text {added }}$ ), compared to non-bioaugmentation $(296 \mathrm{~L} / \mathrm{kg} \mathrm{VS}$ added). The first peak value with bioaugmentation is $717 \mathrm{~L} / \mathrm{kg} \mathrm{VS}$ added followed by second and third peak value $893.88 \mathrm{~L} / \mathrm{kgVS}_{\text {added }}$ and $512.74 \mathrm{~L} / \mathrm{kgVS}_{\text {added, }}$ respectively. This implied that initial microbial consortium was strengthened with bioaugmentation culture (Jiang et al., 2020). Then bioaugmentation shows a positive impact because it can increase the production of the average methane yield from FW is $396 \mathrm{~L} / \mathrm{Kg} \mathrm{VS}$ (Vögeli et al., 2014). Moreover, statistical analysis was showed the sig value. $<0.05$ which indicated that addition of microbial consortium have a significant effect.

Table 2. Statistical Test Results of Biogas Volume and Methane Yield

\begin{tabular}{|l|c|c|c|}
\hline \multirow{2}{*}{ Parameters } & \multicolumn{2}{|c|}{ Normality test Shapiro-Wilk } & Significance test \\
\cline { 2 - 4 } & $\begin{array}{c}\text { Without } \\
\text { Bioaugmentation }\end{array}$ & Bioaugmentation & Wilcoxon \\
\hline Biogas volume & Normal & Non-normal & $<0,05$ \\
\hline Methane yield & Non-normal & Normal & $<0,05$ \\
\hline
\end{tabular}

Reactor performance can be strengthened using the ratio of VFA to total alkalinity (TA). VFA inhibition is an important indicator of AD failure then Alkalinity provides resistance to large changes in $\mathrm{pH}$ (Kainthola et al., 2019). Based on Tab 3, the reactor was in the normal range of alkalinity and VFA. The normal range of TA during AD process is between $1000-5000 \mathrm{mg} \mathrm{CaCO} / \mathrm{L}$ (Tchobanoglous et al., 1993) and the inhibitory effect of VFA starts at a concentration of more than $2000 \mathrm{mg} / \mathrm{L}$ for acetic acid (Rocamora et al., 2020). The stability of the AD process was in the range of the VFA/TA ratio $\leq 0.4$ (Wang et al., 2012). Thus, we demonstrated that the reactor in stable stage with bioaugmentation in the AD process.

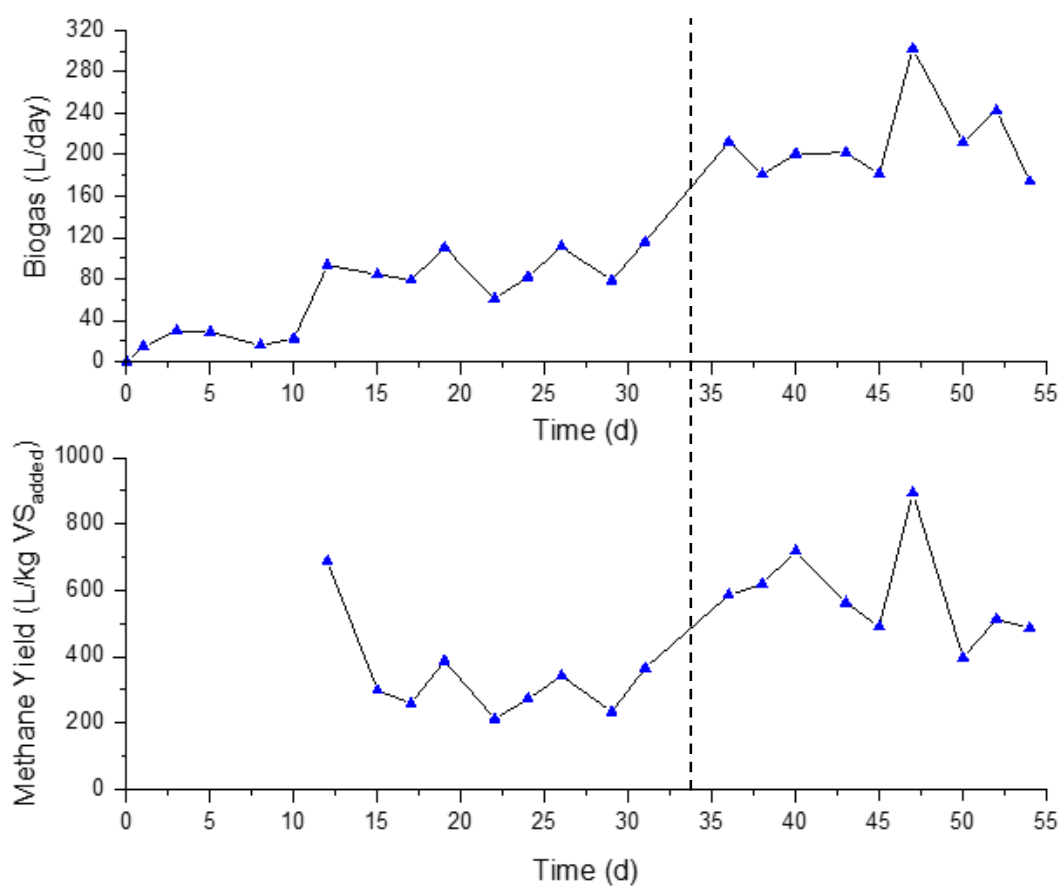




\section{$7^{\text {th }}$ International Conference On}

Modern Approaches in SCIENCE, TECHNOLOGY \& ENGINEERING

\section{8-20 June, 2021}

Brussels, Belgium

Table 3. VFA and Alkalinity Measurement Results in the Reactor

\begin{tabular}{|l|c|c|c|}
\hline \multicolumn{1}{|c|}{ Process } & $\begin{array}{c}\text { Alkalinity } \\
\left(\mathrm{mg} \mathrm{CaCO}_{3} / \mathrm{L}\right)\end{array}$ & $\begin{array}{c}\text { VFA } \\
\left(\mathrm{mg} \mathrm{CH}_{3} \mathrm{COOH} / \mathrm{L}\right)\end{array}$ & VFA/TA \\
\hline $\begin{array}{l}\text { Non- } \\
\text { bioaugmentation }\end{array}$ & 4155 & 577 & 0.1 \\
\hline Bioaugmentation & 3593 & 1061 & 0.3 \\
\hline
\end{tabular}

\subsection{Effect of bioaugmentation on a microbial community}

Analysis of microbial community based on relative abundance is shown in Fig. 2 for top 10 phylum. Firmicutes (45.36\%), Proteobacteria (37.73\%), Actinobacteria $(6.48 \%)$, and Bacteroidetes $(2.43 \%)$ were the dominant bacteria in BS. However, the relative abundance Euryarchaeota as a phylum of archaea is $6.31 \%$. Firmicutes are considered to play a major role in the process of hydrolysis and acidogenesis during the AD process because they could hydrolyze carbohydrates and proteins to produce VFA. Like Firmicutes, Proteobacteria, Actinobacteria, and Bacteroidetes could

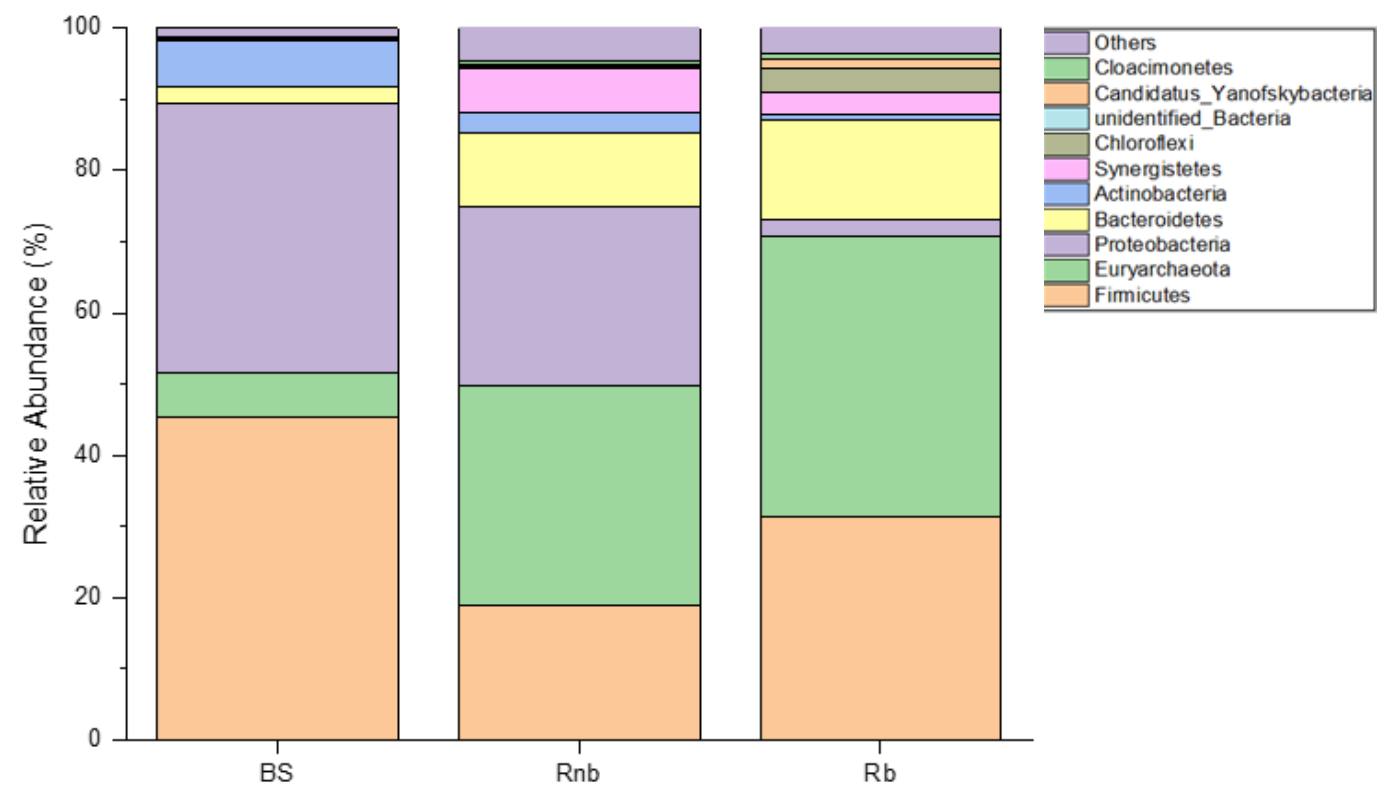

ferment sugars and proteins to produce acetate. Proteobacteria are also capable of degrading lignocellulosic materials in organic waste (Abid et al. 202; Amin et al. 2021). The findings of Firmicutes, Proteobacteria, Actinobacteria, and Bacteroidetes being the dominant phyla in CM are similar to the previous study (Amin et al. 2021).

The relative abundance of archaea as bioaugmentation culture in this study was low compared to several studies using bioaugmentation cultures with higher archaea abundance ( $\mathrm{Li}$ et al., 2018a; Li et al., 2018b; Jiang et al., 2020). The fundamental difference is due to the enrichment of the bioaugmentation culture. Previous studies used a microbial consortium derived from sludge full-scale anaerobic digester so that the relative abundance of archaea was greater, while this study used CM without enrichment that the relative abundance of archaea was $<10 \%$. 


\section{$7^{\text {th }}$ International Conference On}

Modern Approaches in SCIENCE,

TECHNOLOGY \& ENGINEERING

\section{8-20 June, 2021}

Brussels, Belgium

Without bioaugmentation $\left(\mathrm{R}_{\mathrm{nb}}\right)$, Proteobacteria (25.12\%), Firmicutes (19.05\%), Bacteroidetes (10.28\%), Synergistetes (6.21\%), and Actinobacteria (2.98\%) were dominant bacteria. The population of Proteobacteria, Actinobacteria, and Synergistetes were decreased in bioaugmentation $\left(\mathrm{R}_{\mathrm{b}}\right)$ with their relative abundance $2.39 \%, 0.96 \%$, and $3.04 \%$, respectively. However, the relative abundance of Firmicutes and Bacteroidetes increased to $31.37 \%$ and $13.84 \%$, respectively, led to an increase of the VFA concentration. The increase of Firmicutes was caused by the BS that dominated by Firmicutes. Bioaugmentation using the same microbes as the original microbes that have been adapted in the AD reactor can increase their population (Jiang et al., 2020). The increase of Bacteroidetes in the reactor might suggest a preferable degradation tendency for nitrogenous compounds. The decrease of Proteobacteria after bioaugmentation was replaced by Chloroflexi. The Changes in the relative abundance of Chloroflexi are related to the content of biodegradation

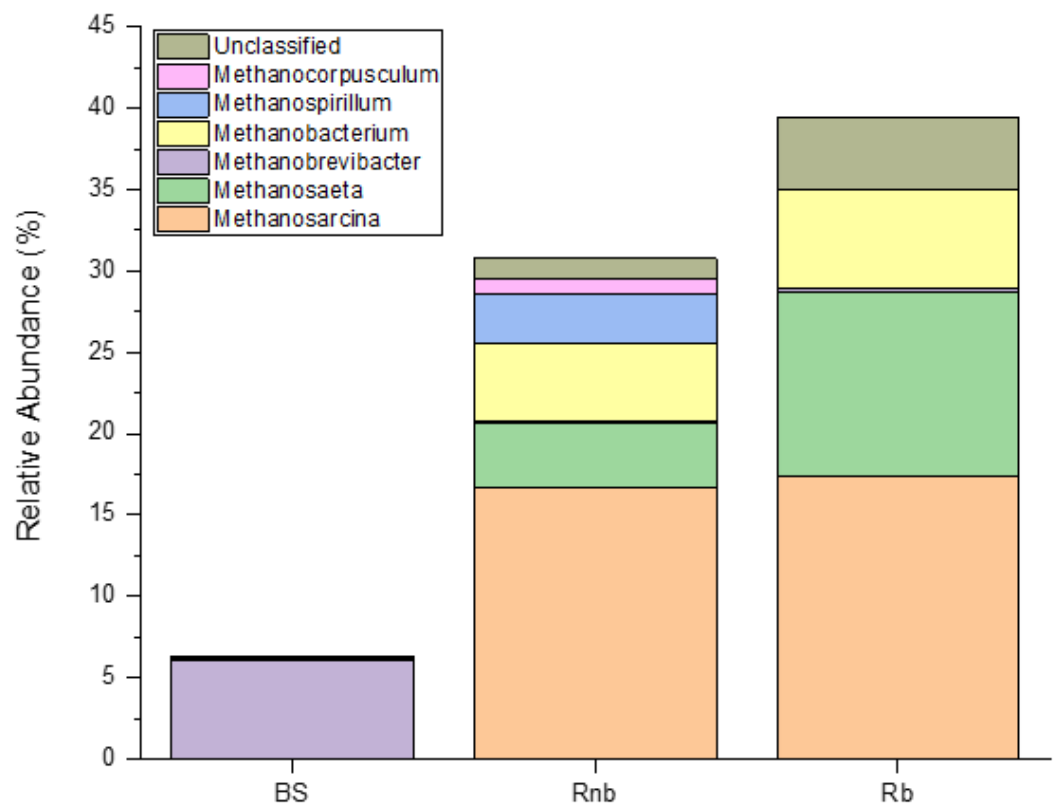

components in which the addition of a microbial consortium from $\mathrm{CM}$ which also contains lignocellulosic material (Li et al., 2020c). Similarly, the predominance of Bacteroidetes, Firmicutes, Proteobacteria, Chloroflexi, Synergistetes, and Actinobacteria has been previously reported in mesophilic AD treating FW (Zhang et al., 2019; Zhang et al., 2018).

Figure 3: Archaea community at genus level 
The comparison of process with and without bioaugmentation shows that the changes in archaea (Fig 3). The percentage increased to 30.77\% - 39.44\%. The genus of domain archaea contained in BS are Methanobrevibacter (6.10\%), Methanobacterium (0.03\%), Methanosarcina (0.01\%), and Methanosaeta $(0.01 \%)$. Methanobrevibacter has been reported to be the dominant archaea in CM (Hagen et al., 2014). Methanobrevibacter and Methanobacterium are common types of methanogens found inside multicellular organisms, namely the digestive tract. The digestive system of animals actively absorbs intermediates from the breakdown of complex organics produced by bacteria therefore these nutrients are only available for a relatively short time. As a result, Methanobrevibacter and Methanobacterium as hydrogenotrophic methanogens grow faster and dominate than methanogens utilizing acetate (Schaechter, 2009).

Methanobrevibacter as dominate in BS not significantly increased in Rb. Without bioaugmentation, the relative abundance of Methanobrevibacter was $0.14 \%$ to $0.22 \%$ by bioaugmentation. Methanobacterium also increased from $4.82 \%$ to $6.07 \%$. Methanobrevibacter and Methanobacterium are a hydrogenotrophic methanogen that could produce methane by reducing $\mathrm{CO}_{2}$ with $\mathrm{H}_{2}$ and strictly anaerobic ( $\mathrm{Yu}$ et al., 2020). The low relative abundance of Methanobrevibacter in the reactor may be possible the low capability to compete with the indigeneous microbes and various abiotic factors (Sharma \& Melkania, 2018). Firmicutes as acetogenic bacteria show a positive correlation with Methanosarcina during the AD process (Abid et al., 2021). It was evidenced by increasing the Methanosarcina shown in Fig. 3 from $16.70 \%$ to $17.39 \%$ with bioaugmentation. Methanosaeta also increased from $3.92 \%$ to $11.29 \%$. The genus Methanosaeta able to use acetate for methanogenesis but unable to utilize use $\mathrm{H}_{2}$ for methane production in AD processes (Muratçobanoğlu et al., 2020). In contrast to Methanosarcina, members of the genus Methanosarcina are capable to growing using one of three methanogenic pathways: acetoclastic, hydrogenotrophic, and methylotrophic (Amin et al., 2021).

Methanosaeta have thin filamented shape and hence makes it more sensitive to toxic materials such as high VFA and ammonia. Whereas Methanosarcina exist in thick clusters providing them a shield against inhibitors hence making them more resistant to instability and even deteriorating AD processes (Abid et al. 2021). This was showed that the abundance of Methanosarcina is greater than Methanosaeta. The enhance of Methanosarcina with bioaugmentation has another advantage in the production of methane because they are allows to be more competitive against environmental stresses (Chen \& Chang, 2020). The small abundance of Methanobrevibacter indicated acetoclastic pathway was dominant in the reactor. It was evidenced by the higher relative abundance of Methanosarcina and Methanosaeta. Unidentified of Methanospirillum and Methanocorpusculum with bioaugmentation toughen dominant acetoclastic pathway. Methanospirillum and Methanocorpusculum are known as hydrogenotrophic methanogens (Amin et al., 2021). Based on the results of this study, we demonstrated that the microbial consortium from $\mathrm{CM}$ without enrichment can be used as a bioaugmentation culture to increase the methanogenic microbial communities in AD of FW by maintaining the suitability of the environmental conditions. 


\section{Conclusion}

This study demonstrated that bioaugmentation using microbial consortium from CM without enrichment can be enhance the biogas production 3-fold and methane yield about $45.8 \%$. After bioaugmentation, the reactor showed improved performance on methanogenesis. Bioaugmentation did not permanently shift digester archaeal community structure but provided reinforcement. Moreover, positive effect of bioaugmentation was showed by increasing the relative abundance of Methanosarcina and Methanosaeta. The higher Methanosarcina and Methanosaeta indicate the acetoclastic pathway was dominant on methanogenesis. The success of bioaugmentation depans on environmental factor and the ability of microbial consortium to compete with initial microbes. Future research to routinely adding bioaugmented cultures is warranted to help make bioaugmentation more stable at pilot scale digester.

\section{Acknowledgment}

This work was supported by Ministry of Research and Technology/National Research and Innovation Agency [No. NKB-277/UN2.RST/HKP.05.00/2021].

\section{References}

Abid, M., Wu, J., Seyedsalehi, M., Hu, Y., \& Tian, G. (2021). Bioresource Technology Novel insights of impacts of solid content on high solid anaerobic digestion of cow manure: Kinetics and microbial community dynamics. Bioresource Technology, 333(March), 125205.

Amin, F. R., Khalid, H., Li, W., Chen, C., \& Liu, G. (2021). Enhanced methane production and energy potential from rice straw by employing microaerobic pretreatment via anaerobic digestion. Journal of Cleaner Production, 296, 126434.

APHA, Standard Methods for the Examination of Water and Waste Water, $23^{\text {rd }}$ Edition, American Public Health Assocoation, Washington D C, 2017.

Aragaw, T., Andargie, M., \& Gessesse, A. (2013). Co-digestion of cattle manure with organic kitchen waste to increase biogas production using rumen fluid as inoculums. International Journal of Physical Sciences, 8(11), 443-450.

Chen, H., \& Chang, S. (2020). Dissecting methanogenesis for temperature-phased anaerobic digestion: Impact of temperature on community structure, correlation, and fate of methanogens. Bioresource Technology, 306(March), 123104.

Dhamodharan, K., Kumar, V., \& Kalamdhad, A. S. (2015). Effect of different livestock dungs as inoculum on food waste anaerobic digestion and its kinetics. Bioresource Technology, 180, 237-241.

Fisgativa, H., Tremier, A., \& Dabert, P. (2016). Characterizing the variability of food waste quality: A need for efficient valorisation through anaerobic digestion. Waste Management, 50, 264-274.

Gong, H., Liu, M., Li, K., Li, C., Xu, G., \& Wang, K. (2020). Optimizing dry anaerobic digestion at pilot scale for start-up strategy and long-term operation: Organic loading rate, temperature and co-digestion. Bioresource Technology, 316(June), 123828. 


\section{8-20 June, 2021 \\ Brussels, Belgium}

Jiang, J., Li, L., Li, Y., He, Y., Wang, C., \& Sun, Y. (2020). Bioaugmentation to enhance anaerobic digestion of food waste: Dosage, frequency and economic analysis. Bioresource Technology, 307(February).

Kainthola, J., Kalamdhad, A. S., \& Goud, V. V. (2019). A review on enhanced biogas production from anaerobic digestion of lignocellulosic biomass by different enhancement techniques. Process Biochemistry, 84(January), 81-90.

Kementerian Lingkungan Hidup dan Kehutanan, Data Persampahan Indonesia 2019, Available: menlhk.go.id.

Li, Ying, Li, L., Sun, Y., \& Yuan, Z. (2018a). Bioaugmentation strategy for enhancing anaerobic digestion of high $\mathrm{C} / \mathrm{N}$ ratio feedstock with methanogenic enrichment culture. Bioresource Technology, 261(2), 188-195.

Li, Ying, Yang, G., Li, L., \& Sun, Y. (2018b). Bioaugmentation for overloaded anaerobic digestion recovery with acid-tolerant methanogenic enrichment. Waste Management, 79, 744-751.

Li, Yu, Zhao, J., Achinas, S., Zhang, Z., Krooneman, J., \& Euverink, G. J. W. (2020c). The biomethanation of cow manure in a continuous anaerobic digester can be boosted via a bioaugmentation culture containing Bathyarchaeota. Science of the Total Environment, 745.

Mehariya, S., Patel, A. K., Obulisamy, P. K., Punniyakotti, E., \& Wong, J. W. C. (2018). Co-digestion of food waste and sewage sludge for methane production: Current status and perspective. Bioresource Technology, 265(March), 519-531.

Negri, C., Ricci, M., Zilio, M., D’Imporzano, G., Qiao, W., Dong, R., \& Adani, F. (2020). Anaerobic digestion of food waste for bio-energy production in China and Southeast Asia: A review. Renewable and Sustainable Energy Reviews, 133(July), 110138.

Oladejo, O. S., Dahunsi, S. O., Adesulu-Dahunsi, A. T., Ojo, S. O., Lawal, A. I., Idowu, E. O., Olanipekun, A. A., Ibikunle, R. A., Osueke, C. O., Ajayi, O. E., Osueke, N., \& Evbuomwan, I. (2020). Energy generation from anaerobic codigestion of food waste, cow dung and piggery dung. Bioresource Technology, 313(June), 123694.

Rocamora, I., Wagland, S. T., Villa, R., Simpson, E. W., Fernández, O., \& BajónFernández, Y. (2020). Dry anaerobic digestion of organic waste: A review of operational parameters and their impact on process performance. Bioresource Technology, 299(December 2019).

Romero-Güiza, M. S., Vila, J., Mata-Alvarez, J., Chimenos, J. M., \& Astals, S. (2016). The role of additives on anaerobic digestion: A review. Renewable and Sustainable Energy Reviews, 58, 1486-1499.

Schanes, K., Dobernig, K., \& Gözet, B. (2018). Food waste matters - A systematic review of household food waste practices and their policy implications. Journal of Cleaner Production, 182, 978-991.

Sharma, P., \& Melkania, U. (2018). Effect of bioaugmentation on hydrogen production from organic fraction of municipal solid waste. International Journal of Hydrogen Energy, 43(15), 7290-7298.

Tchobanoglous, G., Theisen, H. \& Vigil, S., (1993). Integrated Solid Waste Management Enginering Principles and Management Issues. United States: 


\section{$7^{\text {th }}$ International Conference On}

Modern Approaches in SCIENCE,

TECHNOLOGY \& ENGINEERING

18-20 June, 2021

Brussels, Belgium

McGraw-Hill.

Tsapekos, P., Kougias, P. G., Vasileiou, S. A., Treu, L., Campanaro, S., Lyberatos, G., \& Angelidaki, I. (2017). Bioaugmentation with hydrolytic microbes to improve the anaerobic biodegradability of lignocellulosic agricultural residues. Bioresource Technology, 234, 350-359.

Vögeli, Y., Riu, C., Gallardo, A., Diener, S., \& Zurbrügg, C. (2014). Anaerobic Digestion of Biowaste in Developing Countries. In Sandec: Department of Water and Sanitation in Developing Countries.

Wang, L. H., Wang, Q., Cai, W., \& Sun, X. (2012). Influence of mixing proportion on the solid-state anaerobic co-digestion of distiller's grains and food waste. Biosystems Engineering, 112(2), 130-137.

Yu, X., Zhang, C., Qiu, L., Yao, Y., Sun, G., \& Guo, X. (2020). Anaerobic digestion of swine manure using aqueous pyrolysis liquid as an additive. Renewable Energy, 147, 2484-2493.

Zhang, L., Loh, K. C., \& Zhang, J. (2018). Food waste enhanced anaerobic digestion of biologically pretreated yard waste: Analysis of cellulose crystallinity and microbial communities. Waste Management, 79, 109-119.

Zhang, L., Loh, K. C., Zhang, J., Mao, L., Tong, Y. W., Wang, C. H., \& Dai, Y. (2019). Three-stage anaerobic co-digestion of food waste and waste activated sludge: Identifying bacterial and methanogenic archaeal communities and their correlations with performance parameters. Bioresource Technology, 285(February), 121333. 